\title{
Sodium Azide Resistance in Proteus hauseri
}

\author{
By J. N. COETZEE, H. C. DE KLERK AND I. J. MARÉ \\ Department of Microbiology, University of Pretoria, Pretoria, South Africa
}

SUMMARY

The sodium azide resistance pattern of Proteus hauseri is not of the allor-none unilocal type reported for Escherichia coli but rather of the obligatory multi-step or penicillin variety. Independently isolated 1st-step resistant variants possessed similar degrees of resistance to sodium azide. Some properties of azide-resistant variants of $\boldsymbol{P}$. hauseri are described. In support of the above finding it was possible to transduce 1st-step (and only 1st-step) resistance into the wild-type by phage grown on either 1st-, 2nd-, 3rd- or 4th-step resistant organisms. It was also possible to transduce 2 ndstep resistance into 1st-step resistant organisms by phage developed on independently isolated 1st-step resistant organisms or by phage from multi-step resistant variants. About $60 \%$ of transduced genes expressed their phenotype in platings done immediately after the adsorption period. It is concluded that a number of loci, not closely linked, and possibly equipotent, control sodium azide resistance in $P$. hauser $i$ and that resistance could be dominant to the wild allele.

\section{INTRODUCTION}

Three patterns of resistance development by bacteria to inhibitory agents have been observed (Bryson \& Szybalski, 1955). With the obligatory single-step pattern (Manten \& Rowley, 1953) organisms possessing maximal resistance may be selected by a single exposure to the agent. No intermediate degrees of resistance are encountered. Multistep resistance is a gradual step-wise process and requires repeated exposure and selection to obtain organisms with a high degree of resistance. This is the mode of development of resistance to penicillin (Demerec, 1948) and most other antibiotics (Bryson \& Szybalski, 1955). In the facultative single-step pattern survivors possessing all grades of resistance may be selected by a single exposure to the inhibitor. This is the pattern of resistance elicited by streptomycin (Demerec, 1948). The discrete polygenic mechanisms governing the penicillin type of resistance were successfully dissected by the transformation studies of Hotchkiss (1951), the recombinant analysis of Cavalli (1952) and Cavalli \& Maccacaro (1950, 1952), and the transduction studies of Banič (1959). The transformation experiments of Hotchkiss (1952) and the transduction studies of Watanabe \& Watanabe (1959a) also confirmed the facultative one-step pattern governing resistance to streptomycin.

The inhibitory action of sodium azide on bacterial growth was known since the work of Loew (1891). Keilin \& Hartree (1934) demonstrated that sodium azide was an inhibitor of catalase and the differential effect it had against the growth of many Gram-negative organisms as well as aerobes has been exploited in numerous selective media (Forget \& Fredette, 1962). Sodium azide resistance was first mentioned as a marker in bacterial genetics by Lederberg (1947). Lederberg (1950) isolated an azide resistant mutant of Escherichia coli strain w 478 on nutrient agar containing M/500 sodium azide. Cavalli (1952) claimed that sodium azide resistance in micro- 
organisms was of the all-or-none type but also stated that 'azide can perhaps be said to belong to the group of drugs giving the streptomycin pattern of resistance'. Bryson \& Szybalski (1955) stated that resistance to sodium azide was of the obligatory single-step pattern. Neither Cavalli (1952) nor Bryson \& Szybalski (1955) referred to survival studies of micro-organisms exposed to different concentrations of sodium azide to support their claims, but both cautioned that the jump to azide resistance was small. Cavalli \& Maccacaro (1950) and Cavalli (1952) crossed independently isolated azide-resistant mutants of $E$. coli and demonstrated that azide resistance was controlled by a gene locus linked to the threonine and leucine loci. No details regarding the isolation of the mutants were given and the concentration of azide used in the experiments was not stated. The concentration of sodium azide used in bacterial crosses (in various media) to select for the azide resistance marker was never greater than $\mathrm{M} / \mathbf{5 0 0}$, and Hayes (1957) and Jacob \& Wollman (1961) used $\mathrm{M} / \mathbf{1 6 0 0}$ and $\mathrm{M} / 850$ respectively. Bryson \& Szybalski (1955) referred to unsuccessful experiments by P. E. Hartman to transduce azide resistance by means of bacteriophage, but Lennox (1955) was the first to report the transduction of a M/500 azide resistance marker, using phage $\mathbf{P} 1$ in an $\boldsymbol{E}$. coli system. He did not directly select for azide resistance but showed that the marker could be jointly transduced with either the leucine or the arabinose marker. Coetzee \& Sacks (1960 $a$ ) reported the transduction of streptomycin resistance in Proteus mirabilis. An initial failure to transduce resistance to sodium azide stimulated the work reported here.

\section{METHODS}

Media. The composition of the MacConkey-type agar, soft agar and the broth employed are described by Coetzee \& Sachs $(1960 b)$. Sodium azide (British Drug Houses) was added to the sterilized broth or to the molten agar at $47^{\circ}$. Azide media were prepared on the day of use. Dilutions of cultures were made in sterile $0.85 \%$ $(\mathrm{w} / \mathrm{v})$ saline in distilled water. Solid media were inoculated with $0 \cdot 1 \mathrm{ml}$. of dilutions of broth cultures and spread to dryness with a sterile glass rod. McIntosh and Fildes's jars (Mackie \& McCartney, 1960) were used for anaerobic cultures. Cultures were incubated at $37^{\circ}$ for $48 \mathrm{hr}$. unless otherwise stated.

Organisms. Proteus mirabilis strains 13, 193, 57 and phages 34/13, 14/13 str-r 12/57, 12/57 str-r were used throughout. In addition 4 strains of $P$. vulgaris freshly isolated from human faeces were used in survival-curve studies. Strains 13, 193, 57 and the phages have been described as well as the methods used for preparation of high-titre phage stocks and measurement of phage adsorption, and to detect lysogeny in transductants (Coetzee \& Sacks, $1960 a$ ). Variants resistant to sodium azide are identified by $a z-r$ and a number indicating the step of resistance. Thus $13 a z-r 4$ denotes a 4th-step azide resistant variant of strain 13. Catalase activity of cultures was qualitatively tested according to the method of Davis (1955). The flagellar stain and the hanging-drop motility method are described by Mackie \& McCartney (1960). Flagellar antisera were prepared, adsorbed and tested according to Kauffman (1951).

Survival studies. An overnight broth culture of the wild-type organism was concentrated by centrifugations to $c .2 \times 10^{10}$ viable organisms $/ \mathrm{ml}$. and plated on MacConkey agar containing different concentrations of sodium azide. Plates were 
incubated and surviving colonies counted. A colony on the highest concentration of sodium azide was picked into $100 \mathrm{ml}$. broth and incubated overnight. Dilutions of this culture were made and plated on different azide concentrations. After incubation the selection process was repeated.

Growth-rate experiments. These were done by inoculating $c .10^{5}$ organisms contained in $0.1 \mathrm{ml}$. of a dilution of an overnight broth or sodium azide broth culture into $100 \mathrm{ml}$. of broth or azide broth. Cultures were maintained at $137^{\circ}$ in a water bath and sterile air bubbled through them. At intervals, dilutions were plated on plain or azide MacConkey and incubated.

Transduction techniques. Two tubes each containing $1.0 \mathrm{ml}$. of an overnight culture of the recipient organism (about $10^{9}$ viable organisms $/ \mathrm{ml}$.) were centrifuged to clarity and the supernatants decanted. One ml. of transducing phage $\left(6-8 \times 10^{9}\right.$ plaque-forming particles $/ \mathrm{ml}$.) was then added to one of the tubes. One ml. of either phage $34 / 13 s t r-r$ or $12 / 57 s t r-r$ (titre, $6 \times 10^{9}$ plaque-forming particles $/ \mathrm{ml}$.) was added to the other tube which served as the organism control. One ml. of each phage suspension was added to $3 \mathrm{ml}$. of broth in other tubes to confirm the sterility of the stock. After gentle agitation in a water bath at $37^{\circ}$ for $20 \mathrm{~min} .0 \cdot 1 \mathrm{ml}$. from the first two tubes were plated on azide MacConkey and plain MacConkey and incubated. The latter plates were incubated for $5 \mathrm{hr}$. and then carefully overlayed with $4 \mathrm{ml}$. soft agar at $45^{\circ}$ containing sufficient sodium azide to give the desired concentration after diffusion. After the agar had set, the plates were incubated. In certain experiments the contents of the tubes were also titrated on plain MacConkey for viable organisms. Phage sterility controls were plated on MacConkey agar after overnight incubation. The role of phage in lysates which yielded positive results was established according to methods previously used (Coetzee \& Sacks, $1960 a$ ).

\section{RESULTS}

Stability of media. In view of the reports by Pike (1944), Diamond (1950) and Gerencser \& Weaver (1959) on the instability of sodium azide-containing media the following experiments were done. MacConkey azide media were pre-incubated for periods of 1-8 days before inoculation with sensitive and azide resistant variants and further incubated. Simultaneously, freshly prepared azide media were also inoculated and incubated. In a series of three experiments the pre-incubated plates yielded similar counts to those on the freshly prepared azide media and proved their stability under test conditions. Forget \& Fredette (1962), and workers quoted by them, also found azide-containing media (often prepared by more vigorous methods) to be stable for at least 7 days. As a matter of convenience freshly prepared media were nevertheless used for each day's experiments.

Survival in presence of sodium azide. Figure 1 shows a family of survival curves of organism 13 on graded concentrations of sodium azide. This composite presentation shows the resistance pattern of the organism to the particular inhibitory agent (Demerec, 1945, 1948). It is apparent from the survival curve of the wild-type organism that azide resistance is not of the obligatory single-step type (Manten \& Rowley, 1953). Resistance is of the multi-step variety and the fact that the $2 \times 10^{9}$ wild-type organisms plated did not contain variants resistant to the highest concentrations of azide makes the streptomycin or facultative multi-step pattern 
unlikely, and indicates that resistance to azide in this strain is of the obligatory multi-step or penicillin variety (Bryson \& Szybalski, 1955). Variants surviving on м/217 azide were named 1st-step resistants. 2nd-, 3rd-, 4th-step resistant organisms were variants which grew on $\mathrm{M} / 130, \mathrm{M} / 82, \mathrm{M} / 54$ sodium azide respectively. 4th-step resistant variants were not resistant to concentrations much higher than $\mathrm{m} / \mathbf{5 4}$ but selection was not carried further. As in the case of penicillin (Demerec, 1945, 1948), the build-up of resistance was more rapid with each consecutive selection. A number

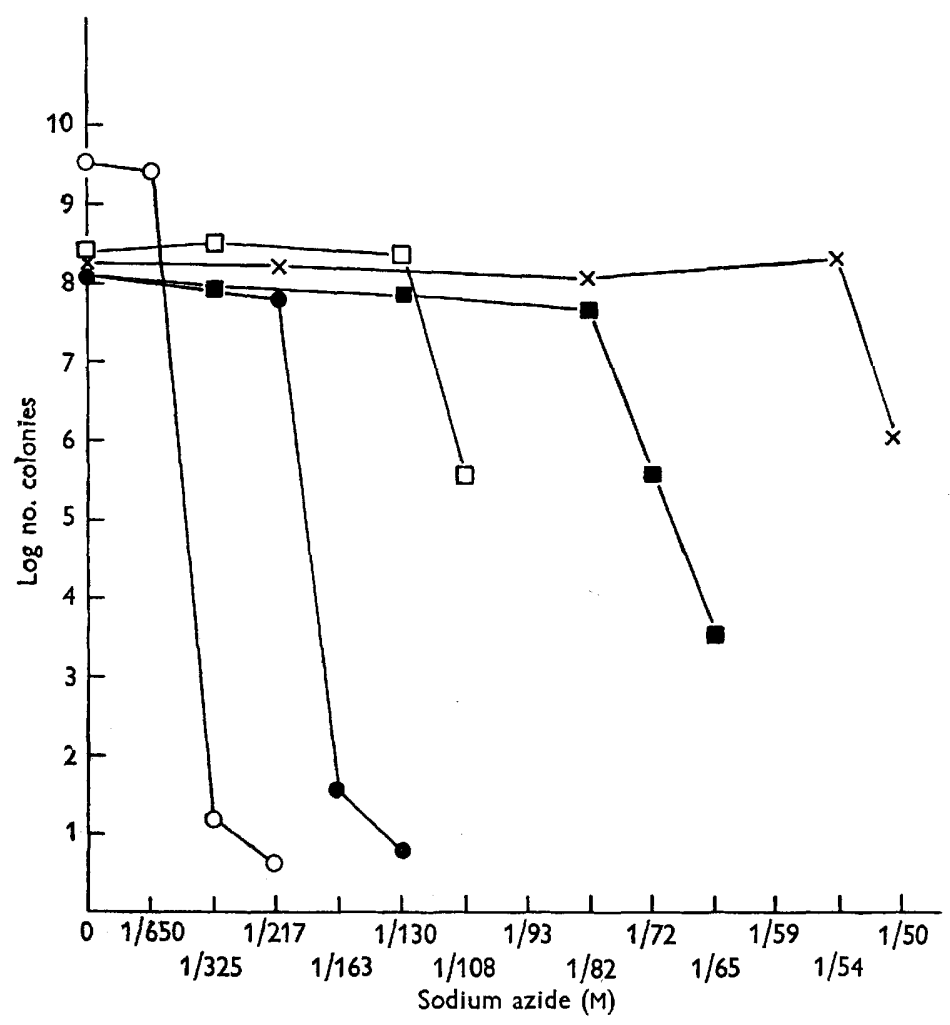

Fig. 1. Growth of Proteus mirabilis strain 13 on graded concentrations of sodium azide. A broth culture of strain 13 was concentrated to $2 \times 10^{10}$ viable organisms $/ \mathrm{ml}$. and $0.1 \mathrm{ml}$. of suitable dilutions plated on different concentrations of sodium azide MacConkey. After $48 \mathrm{hr}$. incubation colonies were counted and one which grew on the highest concentration of azide picked into $100 \mathrm{ml}$. broth and incubated overnight. This culture was then again plated on MacConkey azide and the selection process repeated a number of times. $\bigcirc-O$, Wild type; $-\bigcirc$, Ist-step resistant; $\square-\square$, 2nd-step resistant; a, 3rd-step resistant; $x-x, 4$ th-step resistant variants.

of independently-isolated 1st-step variants of strain 13 had similar ranges of azide resistance. This also applied to 2 nd-, 3rd- and 4th-step resistant variants and is in agreement with the findings of Demerec (1948) for penicillin-resistant mutants of staphylococci. Organism 193 was very sensitive to sodium azide and there were no survivors on $\mathrm{M} / \mathbf{5 0 0}$ azide. Selection, however, yield a family of curves which followed the resistance pattern of the other strains very closely. The four freshly isolated strains of Proteus vulgaris, as well as $\boldsymbol{P}$. mirabilis strain 57, gave similar survival curves. Colonies representative of each resistance step were re-streaked on 
azide media for purification before single colonies were inoculated on nutrient agar slants. After $24 \mathrm{hr}$. incubation they were stored at $4^{\circ}$.

Properties of resistant variants. Resistance to sodium azide appeared to be stable in that stock cultures maintained on nutrient agar and subcultured at about 2-monthly intervals for 2 years maintained their resistant properties. Variants of each degree of resistance produced colonies on media containing the corresponding concentrations of azide with diameters only about two-thirds of those of wild-type colonies on MacConkey agar. There was also a small variation in colony diameter among variants with the same degree of resistance, but no marked difference in colonial morphology was encountered between variants with different degrees of resistance. On plain MacConkey agar, all resistant variants produced colonies similar to but usually very slightly smaller than those of the wild-type. In azide media variants not only had long lag periods but also longer generation times than the wild-type. Similar growth differences from the wild-type were noted by Mitchison (1953) for streptomycin-resistant variants of Escherichia coli and by Mayr-Harting (1955) for a series of penicillin-resistant variants of staphylococci. In plain broth these differences were much smaller and variants and wild-types eventually attained similar maximum viable counts. Drug-resistant organisms are usually more slow-growing than the corresponding sensitive wild-types (Demerec, 1951 ; Michison, 1953; Watanabe, 1954; Mayr-Harting, 1955; Banič, 1959; Thornley $\&$ Yudkin, 1959) and the azide-resistant variants grew slower in the presence of the particular inhibitory agent than in its absence and so resembled the type III $b$ streptomycin-resistant variants of $\boldsymbol{E}$. coli described by Mitchison (1953), some of the Staphylococcus aureus penicillin-resistant strains of Mayr-Harting (1955), and the $\mathrm{M}_{1}$ proflavine-resistant variants of $E$. coli of Thornley \& Yudkin (1959).

Table 1. Experiment to determine the effect of anaerobiosis on azideresistant variants of strain 13

Broth cultures of $13 a z-r 1$ and $13 a z-r 4$ were diluted to $c, 10^{3}$ viable organisms $/ \mathrm{ml}$. and $0.1 \mathrm{ml}$. plated on duplicate plates of azide-MacConkey and plain MacConkey agar. One of the azide and plain MacConkey agar sets were incubated anaerobically for $48 \mathrm{hr}$. The remaining plates were incubated aerobically for $48 \mathrm{hr}$.

No. of colonies on azide-MacConkey agar

\begin{tabular}{|c|c|c|c|c|c|c|c|c|c|c|}
\hline \multirow[b]{2}{*}{ Variant } & \multicolumn{5}{|c|}{ Anaerobic } & \multicolumn{5}{|c|}{ Aerobic } \\
\hline & 0 & $\mathrm{M} / 217$ & M/108 & $M / 82$ & $\mathrm{M} / 54$ & 0 & $\mathrm{M} / 217$ & M/108 & $\mathbf{M} / 82$ & $M / 54$ \\
\hline $13 a z-r 1$ & 243 & 0 & 0 & 0 & 0 & 221 & 234 & o & $\mathbf{0}$ & 0 \\
\hline $13 a z-r 4$ & 190 & 0 & 0 & 0 & 0 & 178 & 200 & 210 & 183 & 181 \\
\hline
\end{tabular}

All resistant variants were motile, flagellated, and agglutinated with pure $\mathbf{H}$ antisera. 1st-, 2nd-, 3rd-step variants also swarmed, particularly at room temperature on MacConkey azide agar. No 4th-step variants were encountered which swarmed on $\mathbf{M} / \mathbf{5 4}$ azide MacConkey although they swarmed on plates containing $\mathbf{M} / 82$ azide. Sodium azide ( $\mathrm{M} / 650$ in blood agar) was used by Snyder \& Lichstein (1940) to inhibit the spreading of Proteus colonies.

Results presented in Table 1 indicate that azide resistant variants were unable to grow anaerobically on azide-containing MacConkey agar though they could do so on the azide-free medium. All azide resistant variants behaved in this manner. All 
the variants produced catalase when growing aerobically on azide-MacConkey or anaerobically on plain MacConkey agar. There are no reports about the respiratory activity of azide-resistant variants, but Sevag \& Shelburne (1942) found that the aerobic respiration of strains of streptococci and pneumococci was strongly inhibited by this chemical. Lichstein \& Soule (1944a) and Forget \& Fredette (1962) demonstrated that anaerobic organisms were more resistant to sodium azide than aerobic organisms. The former workers and Schäfer \& Haas (1957) also observed that a series of Gram-negative bacteria and staphylococci were more sensitive to sodium azide when cultured anaerobically. There are no reports concerning the catalase activity of azide-resistant organisms, but Lichstein \& Soule $(1944 b)$ showed that organisms which lacked catalase were most resistant to azide and these authors as well as Schäfer \& Haas (1957) demonstrated that azide markedly inhibited the catalase activity of bacteria.

Table 2. Transduction experiment with phage 34/13 az-r3 and strain 13 as recipient

$1 \mathrm{ml}$. of phage $34 / 13 \mathrm{az}-\mathrm{r} 3$ (plaque-forming titre: $6 \times 10^{9} / \mathrm{ml}$.) was mixed with a deposit of $10^{9}$ recipient bacteria. $1 \mathrm{ml}$. of phage $34 / 13 \mathrm{str}-r$ with the same titre was added to an equal number of the bacteria as a control. After $20 \mathrm{~min}$. adsorption at $37^{\circ}$, $0.1 \mathrm{ml}$. of each was plated on MacConkey agar and incubated for $5 \mathrm{hr}$. The plates were then overlayed with $4 \mathrm{ml}$. soft agar containing sodium azide to give the desired concentrations after diffusion. Plates were then incubated for $48 \mathrm{hr}$. and the colonies counted. Phage controls were sterile.

Concentration of sodium azide in MacConkey agar

\begin{tabular}{|c|c|c|c|c|c|}
\hline & $\mathbf{M} / 500$ & $\mathrm{M} / \mathbf{3 2 5}$ & $\begin{array}{c}\mathrm{M} / 217 \\
\text { No. of colonies }\end{array}$ & $\mathrm{M} / \mathbf{1 6 3}$ & $\mathrm{M} / \mathbf{1 3 0}$ \\
\hline Test & + & 294 & 333 & 9 & 0 \\
\hline Control & + & 11 & 2 & 0 & 0 \\
\hline
\end{tabular}

Transduction experiments. Table 2 shows results of an experiment to transduce 3rd-step azide resistance into the wild strain 13 with phage 34/13 az-r3. The phage controls in this and other experiments reported were sterile. The test plates had numerous colonies growing on $\mathbf{M} / \mathbf{2 1 7}$ azide while the controls infected with phage 34/13 str-r had few colonies on concentrations above M/500. A further experiment with the same lysate but with 13 replaced as recipient by the wild-type strain 193 yielded even more clear-cut results in that the test had many colonies on $\mathbf{M} / 217$, whereas the $\mathbf{M} / \mathbf{5 0 0}$ control plates were barren. The transducing ability of the lysate was not affected by pre-treatment with deoxyribonuclease but transduction was completely abolished by the addition of a small volume of high-titre anti-phage serum which reduced the plaque-forming titre of the lysate to less than $10^{5} / \mathrm{ml}$. These experiments established the phage in the lysate as the genetic vector. It appeared that a phage lysate of $13 a z-r 3$ could only transduce wild-type strains to $\mathrm{M} / \mathbf{2 1 7}$ (i.e. 1st-step) azide resistance. Experiments were then done with the same recipients, but lysates prepared on variants $13 a z-r 1,13 a z-r 2$, and $13 a z-r 4$. Again only 1st-step resistance was transduced. Table 3 shows an experiment in which variant 193 az-r 1 was treated with a phage lysate of $13 a z-r 1$. The test plates had numerous colonies on $\mathbf{M} / \mathbf{1 2 0}$ while the control had few colonies above a concentration of $\mathbf{M} / 217$. This 
result was taken to mean that a 1st-step resistant variant could be transduced to 2nd-step resistance by a lysate prepared on an independently isolated 1st-step variant. In a further experiment variant $13 a z-r 1$ was treated with the same lysate used in the first experiment reported-i.e. phage 34/13 $a z-r 3$. The control counts were 9 colonies on $\mathrm{M} / 163$ and 0 on $\mathrm{M} / 120$. The test had 217 and 196 colonies on these concentrations respectively. There was thus the situation where lysate $13 a z-r 3$ could transduce no more than 1st-step resistance in wild strains but could produce 2nd-step resistance from a 1st-step resistant organism. Similar results were obtained with Proteus mirabilis strain 57 and phage $12 / 57$ system. In both systems it was possible to transduce 3rd-step sodium azide resistance into 2 nd-step variants with lysates prepared on 4th-step resistant variants. Adsorption experiments showed that at least $95 \%$ of the phage adsorbed within the 20 min. adsorption period. On this basis the transduction rate was about $10^{-7} /$ phage particle adsorbed. In all

\section{Table 3. Transduction experiment with phage 34/13 az-r1 and variant 193 az-r1} as recipient

$1 \mathrm{ml}$. of phage $34 / 13 a z-r 1$ (plaque-forming titre: $8 \times 10^{9} / \mathrm{ml}$.) was mixed with a deposit of $1 \times 10^{9}$ recipient bacteria. $1 \mathrm{ml}$. of phage $34 / 13 \mathrm{str}-r$ (plaque-forming titre: $6 \times 10^{2} / \mathrm{ml}$.) was added to an equal no. of the bacteria as a control. After $20 \mathrm{~min}$. adsorption at $37^{\circ}$ $0.1 \mathrm{ml}$. of each was plated on MacConkey agar and incubated for $5 \mathrm{hr}$. The plates were then overlayed with $4 \mathrm{ml}$. soft agar containing sodium azide to give the desired concentrations after diffusion. Plates were then incubated for $48 \mathrm{hr}$. and the colonies counted. Phage controls were sterile.

\begin{tabular}{|c|c|c|c|c|c|c|}
\hline & \multicolumn{6}{|c|}{ Concentration of azide in MacConkey agar } \\
\hline & $\mathrm{M} / 217$ & $\mathrm{M} / 163$ & $\mathbf{M} / \mathbf{1 3 0}$ & $\mathrm{M} / 120$ & $\mathrm{M} / 108$ & $M / 82$ \\
\hline \multirow{3}{*}{$\begin{array}{l}\text { Test } \\
\text { Control }\end{array}$} & \multicolumn{6}{|c|}{ No. of colonies } \\
\hline & + & 325 & 296 & 305 & 15 & 0 \\
\hline & + & 8 & $\mathbf{0}$ & 0 & 0 & $\mathbf{0}$ \\
\hline
\end{tabular}

transduction experiments the number of transductants present on the directly inoculated azide agar was two-thirds of that on the double-agar-layer plates. Figure 2 shows that while about $60 \%$ of transduced $a z-r$ genes were immediately expressed, the greatest number of transductants was obtained by layering with azide-agar 4 to $5 \mathrm{hr}$. after plating. Reconstruction experiments with known numbers of azide-resistant organisms proved that resistant clones were not disturbed by overlaying with the soft agar (Coetzee \& Sacks, 1960a). These experiments were verified by others in which transduction mixtures were plated on azide-MacConkey at intervals and also titrated for total viable counts. Although transductants were present on the earliest platings their numbers showed a disproportionate increase with the 4-5 hr. platings. No abortive transductants (Stocker, 1956; Ozeki, 1956) were encountered. All transductant clones tested proved to be lysogenic but no precautions (Coetzee \& Sacks, 1960a) were taken to prevent secondary lysogenization occurring on the plates. Transductants did not adsorb transducing phage. This was attributed to lysogenic conversion (Coetzee, 1961). Transductants resembled the selected resistant variants in all other respects and have retained their resistance to sodium azide for more than a year.

Failure to appreciate the multi-step nature of sodium azide resistance in Proteus 
mirabilis could account for our initial failure to transduce azide resistance: lysates of highly resistant variants were applied to wild-type recipient strains and selection was for transductants with equivalent resistance.

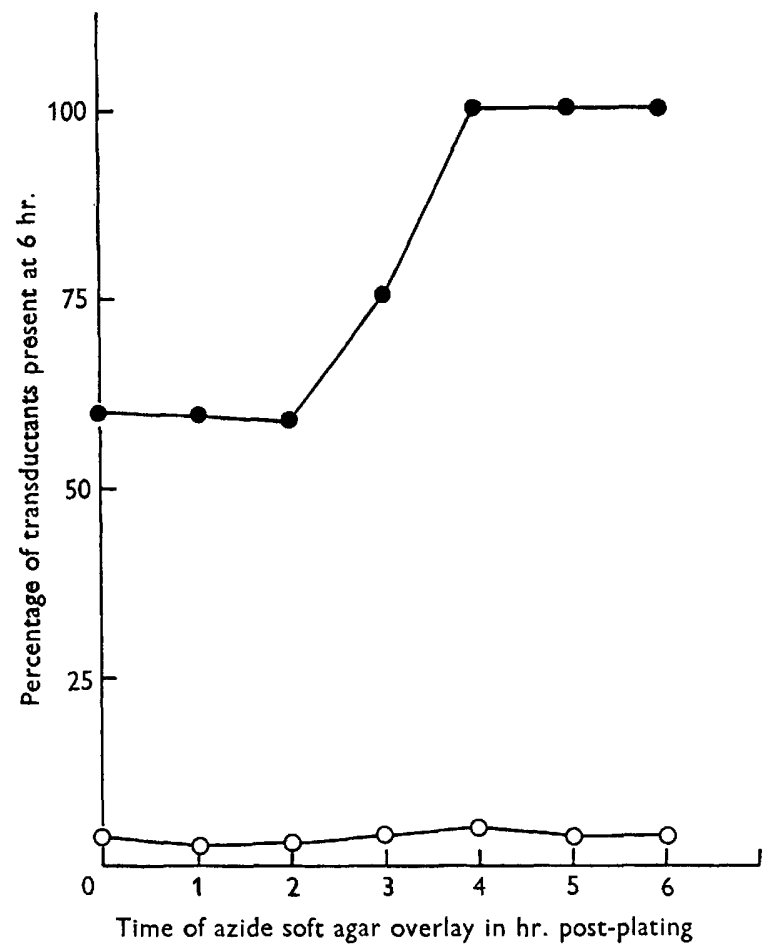

Fig. 2. Phenotypic expression of transduced genes. A transduction experiment was done with strain 57 and phage 12/57 az-r4. Phage 12/57 str-r was added to the organism control. After an adsorption period of $20 \mathrm{~min}$. at $37^{\circ}, 0.1 \mathrm{ml}$. of the test and control were spread on plain MacConkey. The plates were incubated. At intervals they were overlayed with $4 \mathrm{ml}$. of soft sodium azide-containing agar to give a final concentration of $\mathrm{M} / 217$ throughout. After further incubation for $48 \mathrm{hr}$. colonies were counted. The phage controls were sterile.

-O, test; $\mathrm{O}-\mathrm{O}$, control.

\section{DISCUSSION}

The resistance pattern of an organism to an antibacterial agent is a property of the particular agent (Bryson \& Demerec, 1950). Szybalski \& Bryson (1954) encountered an exception. Mycobacterium ranae may become completely resistant to chloramphenicol in a single step, whereas the resistance-pattern of other organisms to this agent is of the multi-step (penicillin) variety. Sodium azide resistance appears to be another exception. In Escherichia coli resistance to this agent is accepted as being obligatory single-step in nature (Cavalli, 1952 ; Bryson \& Szybalski, 1955), while in Proteus hauseri it appears to follow a multi-step pattern. It was possible to transduce 1st-step (and only 1st-step) resistance into wild-type strains by phage grown on 1st- or multi-step resistant variants. 2nd-step resistance was transduced to 1st-step variants by phage produced on independently isolated 1stor multi-step resistant organisms. 1st-step variants were fairly uniform in their degree of resistance to sodium azide. This is what would be expected if resistance to 
sodium azide were governed by a number of more or less equipotent genes not closely enough linked to be jointly transduced (Banič, 1959). Due to lysogenic conversion (Coetzee, 1961), which prevented readsorption of transducing phage it was not possible to attempt the step-wise build-up of resistance to sodium azide by serial re-exposure of transductants to transducing phage as was done in transformation studies (Hotchkiss, 1951). The fact that resistant variants form colonies of practically the same size as the sensitive wild-type on plain MacConkey agar precluded the possibility of transduction of azide sensitivity into resistant variants as achieved by Watanabe \& Watanabe $(1959 b)$ with a str-s marker in Salmonella typhimurium.

The four newly isolated strains of Proteus vulgaris gave sets of survival curves similar to those of $P$. mirabilis strains 13, 913, 57. No transducing phage was available to confirm a polygenic mechanism of azide resistance in the $P$. vulgaris strains, but there is no reason to suppose that they differ from $\boldsymbol{P}$. mirabilis.

In the analysis of phenotypic expression of sodium azide resistance after conjugation in Escherichia coli, Hayes (1957) found that resistance was expressed within the zygotes after a lag of a few minutes and that expression was achieved in the whole population before the recombinants divided. He concluded that resistance to sodium azide was a dominant character in $\boldsymbol{E}$. coli. In the present case it was found that $60 \%$ of transduced resistant genes gained expression on platings done immediately after the adsorption period. The delayed expression of the remaining genes is difficult to explain. Morse (1959) met a similar situation with the transduction of Novobiocin resistance in staphylococci. Following his tentative explanation, it could be argued that, if azide resistance was dominant, the transduced genetic material could require a number of hours to penetrate the recipient organism and so explain the delay in expression. Alternatively, if the azide resistance gene requires a long time for phenotypic expression, it is possible that sodium azide might be slow acting. The transduced resistance genes would then find expression before the organism was inhibited, and so account for the transductants present on early platings. Mutant alleles are usually recessive to the wildtype (Lederberg, 1951; Watanabe \& Watanabe, 1959 $a$; Coetzee \& Sacks, 1960a). The striking exception is the conclusive experiments of Hayes (1957) concerning sodium azide resistance in Escherichia coli. It is tempting to conclude that sodium azide resistance in Proteus mirabilis is also dominant but no decision can be made. The fact that no abortive transductants were observed could point to the recessive nature of azide resistance loci (Watanabe \& Watanabe, 1959 $a$; Coetzee \& Sacks, $1960 \mathrm{a}$ ). However, the absence of abortive transductants in various systems is not unknown (Holloway \& Monk, 1959 ; Edgar \& Stocker, 1961; Thorne, 1962) and they were also not encountered in transduction of the swarming characteristic in Proteus mirabilis (Coetzee, 1963).

Acknowledgement is made to Mrs C. J. Boshoff and Miss C. A. Ferreira for technical assistance rendered. The senior author is in receipt of a grant from the South African Council for Scientific and Industrial Research. 


\section{REFERENCES}

BANič, S. (1959). Transduction to penicillin and chloramphenicol resistance in Salmonella typhimurium. Genetics, 44, 449.

Bryson, V. \& Demerec, M. (1950). Patterns of resistance to antimicrobial agents. Ann. N.Y. Acad. Sci. 53, 283.

Bryson, V. \& Szybalski, W. (1955). Microbial drug resistance. Advanc. Genet. 7, 1.

Cavalli, L. L. (1952). Genetic analysis of drug-resistance. Bull. Wld Hlth Org. 6, 185.

Cavali, L. L. \& Maccacaro, G. A. (1950). Chloromycetin resistance in Escherichia coli, a case of quantitative inheritance in bacteria. Nature, Lond. 166, 991.

Cavalli, L. L. \& Maccacaro, G. A. (1952). Polygenic inheritance of drug-resistance in the bacterium Escherichia coli. Heredity, 6, 311.

Coetzee, J. N. (1961). Lysogenic conversion in the genus Proteus. Nature, Lond. 189, 946.

Coetzee, J. N. (1963). Transduction of swarming in Proteus mirabilis. J. gen. Microbiol. 33, 1.

Coetzee, J. N. \& Sacks, T. G. (1960a). Transduction of streptomycin resistance in Proteus mirabilis. J. gen. Microbiol. 23, 445.

Coetzee, J. N. \& SAcks, T. G. (1960b). Morphological variants of Proteus hauseri. J. gen. Microbiol. 23, 209.

Davis, G. H. G. (1955). The classification of Lactobacilli from the human mouth. J. gen. Microbiol. 13, 481.

Demerec, M. (1945). Production of staphylococcus strains resistant to various concentrations of penicillin. Proc. nat. Acad. Sci., Wash. 31, 16.

Demerec, M. (1948). Origin of bacterial resistance to antibiotics. J. Bact. 56, 63.

Demerec, M. (1951). Studies of the streptomycin-resistance system of mutations in Escherichia coli. Genetics, 36, 585.

Diamond, B. E. (1950). A selective medium for lactobacilli counts from saliva. J. dent. Res. 29, 8.

Edgar, J. B. \& Stocker, B. A. D. (1961). Metabolic and genetic investigations of nutritionally exacting strains of Staphylococcus pyogenes. Nature, Lond. 191, 1121.

Forget, A. \& Fredette, V. (1962). Sodium azide selective medium for the primary isolation of anaerobic bacteria. J. Bact. 83, 1217.

Gerencser, V. F. \& Weaver, R. H. (1959). A new technique for the use of sodium azide (Hydrazoic acid) as an inhibitive agent. Appl. Microbiol. 7, 113.

Hayes, W. (1957). The kinetics of the mating process in Escherichia coli. J. gen. Microbiol. $16,97$.

Holloway, B. W. \& Monk, M. (1959). Transduction in Pseudomonas aeruginosa. Nature, Lond. 184, 1426.

Hотснкіss, R. D. (1951). Transfer of penicillin resistance in pneumococci by the desoxyribonucleate derived from resistant cultures. Cold Spr. Harb. Symp. quant. Biol. 16, 457.

Hотснкіss, R. D. (1952). The role of desoxyribonucleates in bacterial transformations. In Phosphorus Metabolism, Vol. II, p. 426. Ed. W. D. MeElroy and B. Glass. Baltimore: The Johns Hopkins Press.

JaCOB, F. \& Wollman, E. L. (1961). Sexuality and the Genetics of Bacteria. London: Academic Press.

Kauffmann, F. (1951). Enterobacteriaceae. Copenhagen: Einar Munksgaard.

Keilin, D. \& Hartree, E. F. (1934). Inhibitors of catalase reaction. Nature, Lond. 134, 933.

Lederberg, J. (1947). Gene recombination and linked segregations in Escherichia coli. Genetics, 32, 505.

LEDERBERG, J. (1950). The selection of genetic recombinations with bacterial growth inhibitors. J. Bact. 59, 211.

Lederberg, J. (1951). Streptomycin resistance: a genetically recessive mutation. J. Bact. 75, 11. 
Lennox, E. S. (1955). Transduction of linked genetic characters of the host by bacteriophage P1. Virology, 1, 190.

Lichstein, H. C. \& Soule, M. H. (1944a). Studies of the effect of sodium azide on microbic growth and respiration. I. The action of sodium azide on microbic growth. J. Bact. 47, 221.

Lichstein, H. C. \& Soule, M. H. (1944b). Studies of the effect of sodium azide on microbic growth and respiration. II. The action of sodium azide on bacterial catalase. J. Bact. 47, 231.

Loww, O. (1891). Ueber das Verhalten des Aziomids zu lebenden Organismen. Ber. dtsch. chem. Ges. 24, 2947.

Mackie and McCartney's Handbook of Bacteriology (1960). 10th ed. Ed. by R. Cruickshank. Edinburgh: E. and S. Livingstone.

Manten, A. \& Rowlex, D. (1953). Genetic analysis of valine inhibition in the K12 strain of Bacterium coli. J. gen. Microbiol. 9, 226.

MaYR-Harting, A. (1955). The acquisition of penicillin resistance by Staphylococcus aureus strain Oxford. J. gen. Microbiol. 13, 9.

Mitchison, D. A. (1953). The occurrence of independent mutations to different types of streptomycin resistance in Bacterium coli. J. gen. Microbiol. 8, 168.

Monse, M. L. (1959). Transduction by staphylococcal bacteriophage. Proc. nat. Acad. Sci., Wash. 45, 722.

Ozeki, H. (1956). Abortive transduction in purine-requiring mutants of Salmonella typhimurium. Genetic studies with bacteria. Publ. Carneg. Instn, No. 612, p. 97.

Pike, R. M. (1944). An enrichment broth for isolating streptococci from throat swabs. Proc. Soc. exp. Biol., N.Y. 57, 186.

SCHÄFER, W. \& HAAS, F. (1957). Über die Wirkung von Natriumazid auf die Bakterienatmung. Zbl. Bakt. (Abt. 1 Orig.), 169, 402.

Sevag, M. G. \& Shelbourne, M. (1942). Cyanide-sensitive bacterial respiratory systems different from the usual cytochrome-cytochrome oxidase system. J. gen. Physiol. 26, 1.

SNYDer, M. L. \& Lichstein, H. C. (1940). Sodium azide as an inhibitory substance for Gram-negative bacteria. J. infect. Dis. 67, 113.

Stocker, B. A. D. (1956). Abortive transduction of motility in Salmonella; a nonreplicated gene transmitted through many generations to a single descendant. J. gen . Microbiol. 15, 575.

SzYbalski, W. \& Bryson, V. (1954). Genetic studies on microbial cross resistance to toxic agents. III. Cross resistance of Mycobacterium ranae to twenty-eight antimicrobial agents. Amer. rev. Tuberc. 69, 267.

Thorne, C. B. (1962). Transduction in Bacillus subtilis. J. Bact. 83, 106.

Thornley, M. J. \& Yudkin, J. (1959). The origin of bacterial resistance to proflavine. 2. Spontaneous mutation to proflavine resistance in Escherichia coli. J. gen. Microbiol. 20,365.

Watanabe, T. (1954). Genetic studies on the mechanisms of acquired streptomycin resistance in microorganisms. Keio J. Med. 3, 193.

Watanabe, T. \& Watanabe, M. (1959 $a)$. Transduction of streptomycin resistance in Salmonella typhimurium. J. gen. Microbiol. 21, 16.

Watanabe, T. \& Watanabe, M. (1959b). Transduction of streptomycin sensitivity into resistant mutants of Salmonella typhimurium. J. gen. Microbiol. 21, 30. 